\title{
Mefloquine dangers - fact or fancy?
}

I N RECENT MONTHS THERE HAS BEEN A GREAT DEAL OF MISINFORmation concerning the adverse effects of mefloquine after a Canadian military physician suggested that the torture and murder of a Somalian by several Canadian paratroopers might have been related to mefloquine used for malaria chemoprophylaxis.

Severe adverse reactions to mefloquine, namely seizures and psychosis, are rare when the drug is used for prophylaxis, occurring in 1 in 10,000 to 1 in 13,000 users (1). However, when the drug is used for treatment of malaria, such reactions increase to 1 in 215 to 1 in 1700 users (2). In a large retrospective study of more than 100,000 travellers who used antimalarials, side effects from mefloquine occurred in $24 \%$ of users compared with $35 \%$ for chloroquine and proguanil (3). Overall, only about $1 \%$ of mefloquine users had to discontinue prophylaxis because of adverse events due to the drug.

The minor neuropsychiatric adverse effects were recently studied in an excellent randomized, double-blind, controlled trial in American troops in Hawaii (4). There were no statistically significant differences between the side effects due to mefloquine and those due to chloroquine. However, depressive feelings were two to three times more common in the mefloquine group early in the course of the study, and resolved in the majority of subjects as tolerance developed. This reduction in side effects over time has been confirmed in a long term study of Peace Corps volunteers (5).

One of the concerns about long term mefloquine use was the fear of late toxicity due to accumulation of the drug. However, recent pharmacokinetic studies have shown that the steady-state concentration of mefloquine was achieved by the eighth to ninth week of prophylaxis (6). Despite its long elimination half-life, weekly administration did not lead to toxic accumulation of the drug.

One of the main arguments against the use of mefloquine has been the large number of contraindications to the drug. There is little evidence to support contraindications other than a history of seizures or psychosis and presence of cardiac conduction abnormalities. Except for a single anecdotal case, there are no data to support the previous contraindication of mefloquine use in conjunction with beta- and calcium channel blockers. With respect to the contraindication for airline pilots, the study from Boudreau et al (4) in Hawaii and a recent unpublished study of airline pilots in flight simulators showed no change in fine motor coordination or judgement. Finally, the contraindication of mefloquine use in the latter half of pregnancy has been refuted by studies in Malawi on over 2000 women and in a recent placebo controlled trial in Thai women; both studies showed no significant adverse outcome with mefloquine use $(7,8)$. Malaria experts at the Centers for Disease Control and Prevention in Atlanta, Georgia suggest that mefloquine can be considered for prophylaxis of women in their second and third trimester of pregnancy where exposure to chloroquine-resistant Plasmodium falciparum is unavoidable. Since mefloquine has been shown to be safe in the latter half of pregnancy it is very difficult to argue that infants weighing less than $15 \mathrm{~kg}$ should not be given the drug for prophylaxis. Unfortunately, there are no prophylaxis studies of mefloquine use in infancy.

Mefloquine is more efficacious, has similar tolerability and few absolute contraindications relative to other drugs recommended for malaria chemoprophylaxis. Except for those travelling to areas of Southeast Asia where multidrug-resistant malaria is present, mefloquine remains the drug of choice for travellers to all other drug resistant areas of the world.

JS Keystone MD K Kain MD Tropical Disease Unit The Toronto Hospital Toronto, Ontario

\section{REFERENCES}

1. Steffen $R$, Heusser R, Mächler R, et al. Malaria chemoprophylaxis among European tourists in tropical Africa: use, adverse reactions and efficacy. Bull WHO 1990;68:313-22.

2. Weinke T, Trautmann M, Held T, et al. Neuropsychiatric side effects after the use of mefloquine. Am J Trop Med Hyg 1991;45:86-91.

3. Steffen R, Fuchs E, Schildknecht J, et al. Mefloquine compared with other malaria chemoprophylactic regimens in tourists visiting East Africa. Lancet 1993;341:1299-303.

4. Boudreau E, Schuster B, Sanchez J, et al. Tolerability of prophylactic Lariam $®$ regimens. Trop Med Parasitol 1993;44;257-65.

5. Lobel H, Miani M, Eng T. Long-term malaria prophylaxis with weekly mefloquine. Lancet 1993;341:848-51. 
6. Pennie RA, Koren G, Crevoisier C. Steady state pharmacokinetics of mefloquine in long-term travellers. Trans $\mathrm{R}$ Soc Trop Med Hyg 1993;87:459-62.

7. Steketee RW, Wirima JJ. Effective chemoprophylaxis in pregnancy with mefloquine. 39th Annual Meeting of the
American Society of Tropical Medicine and Hygiene, New Orleans, November 4-8,1990. (Abst 196)

8. Nosten F, ter Kuile F, Maelankiri L, et al. Mefloquine prophylaxis prevents malaria during pregnancy: a double-blind, placebo-controlled trial. J Infect Dis 1994;169:595-603. 


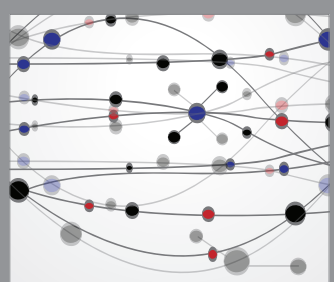

The Scientific World Journal
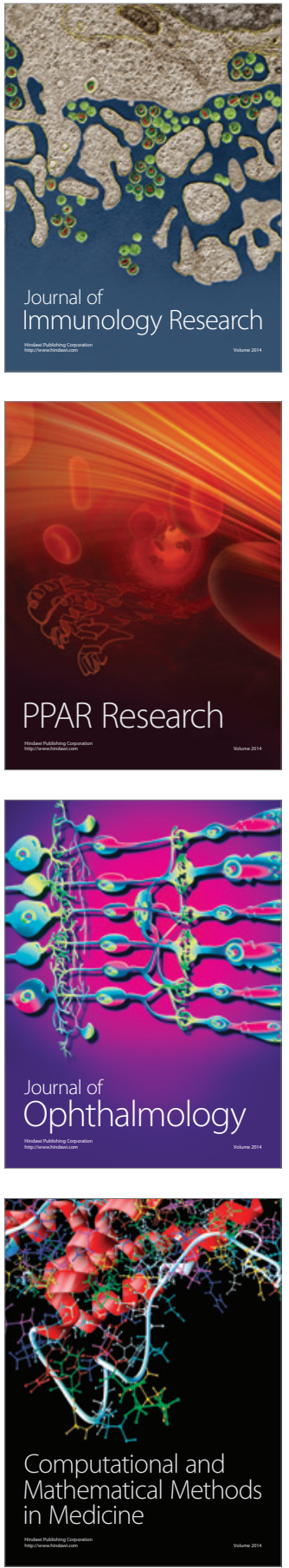

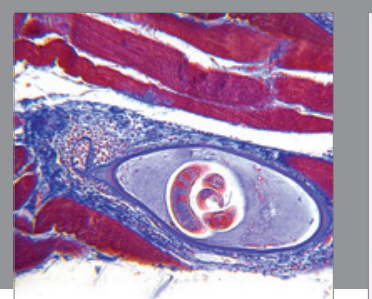

Gastroenterology Research and Practice

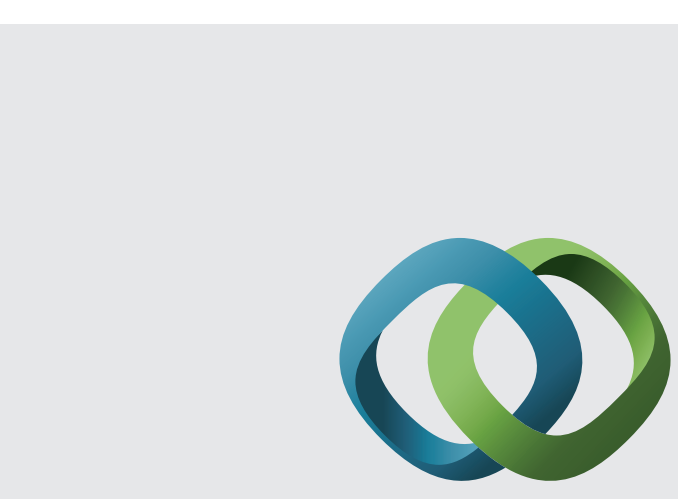

\section{Hindawi}

Submit your manuscripts at

http://www.hindawi.com
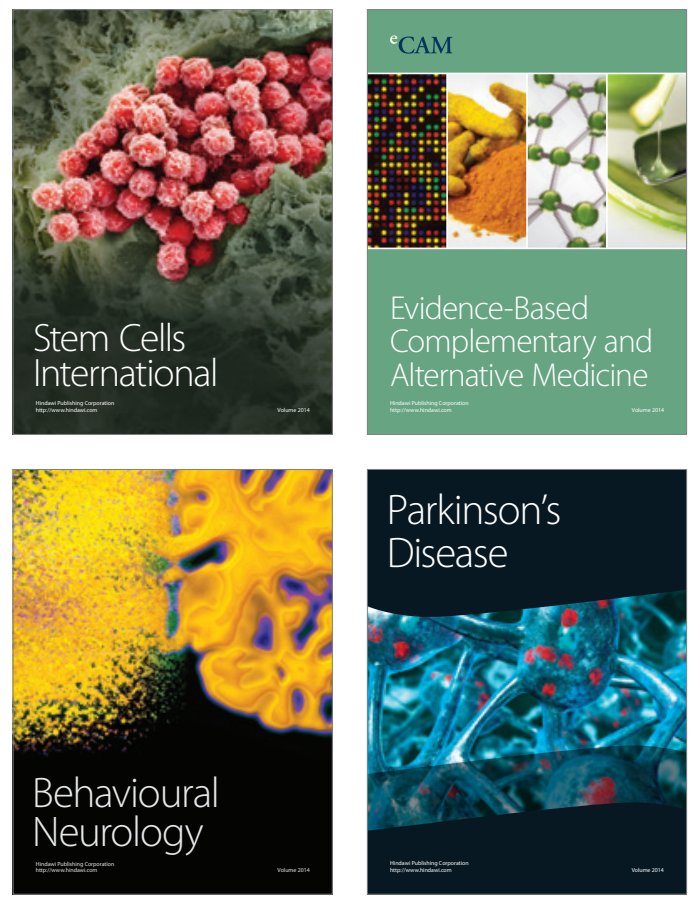
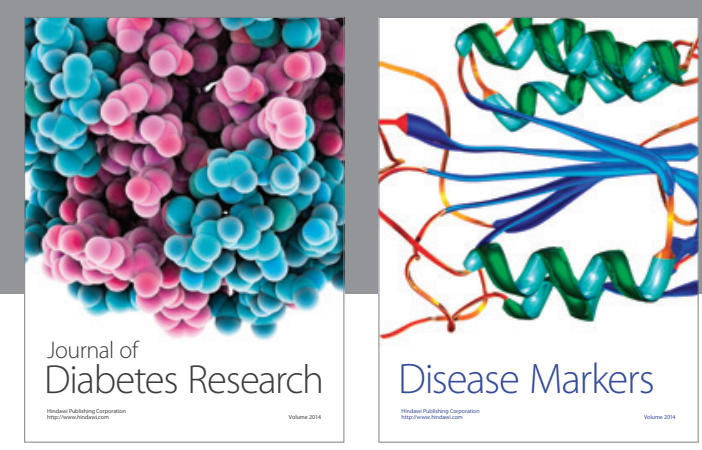

Disease Markers
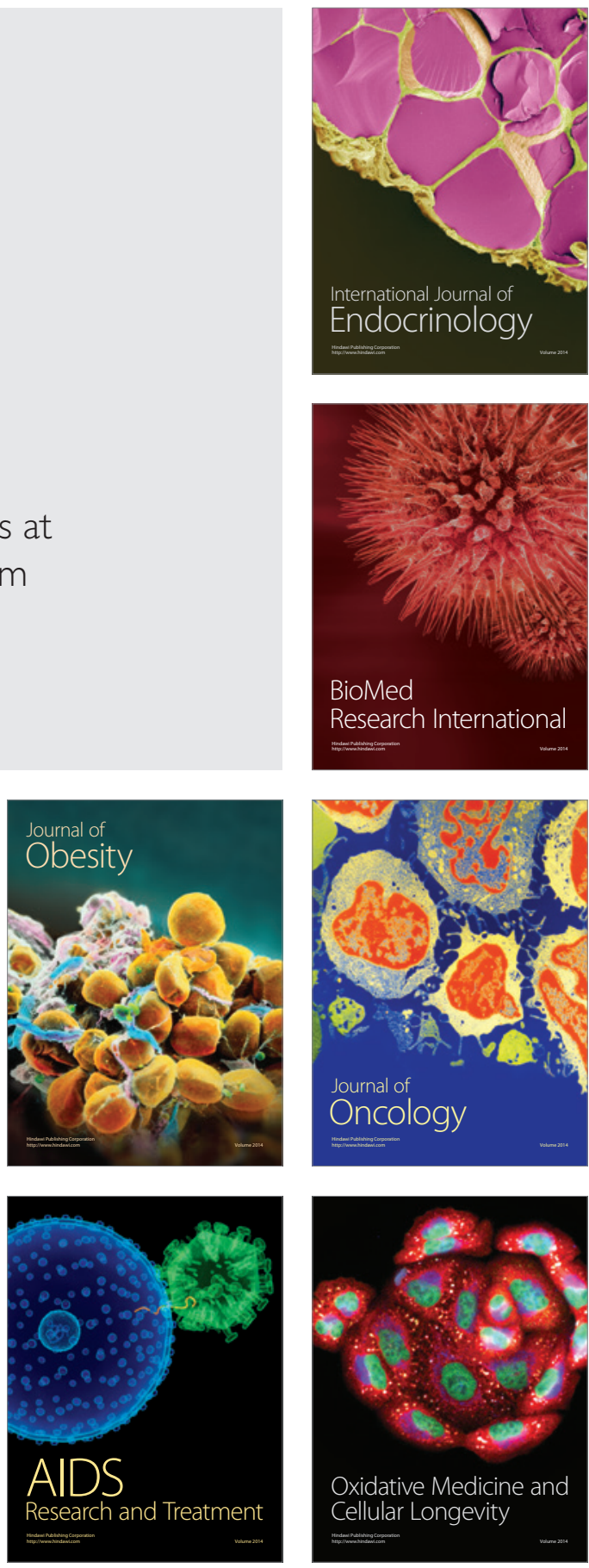